'Servicio de Medicina Interna, Hospital Militar de Santiago, Chile. ${ }^{2}$ Servicio de Cardiología, Hospital Militar de Santiago, Chile.

Los autores declaran no tener conflictos de interés. Trabajo no recibió financiamiento.

Recibido el 26 de abril de 2020 Aceptado el 3 de septiembre de 2020.

Correspondencia a: Dr. José Pardo G. Servicio de Cardiología. Hospital Militar de Santiago, Av. Larraín 9100, La Reina. joseapardo@hotmail.com

\section{Importancia del electrocardiograma en el diagnóstico del infarto agudo al miocardio por obstrucción del tronco común de arteria coronaria izquierda}

\author{
FRANCISCA GONZÁLEZ ${ }^{1}$, MARÍA IGNACIA ARTEAGA ${ }^{1}$, \\ MARÍA LORENA JOFRÉ ${ }^{2}$, MARTIN VALDEBENITO ${ }^{2}$, JOSÉ PARDO²
}

\section{Acute myocardial infarction caused by thrombosis of left main coronary artery. Report of two cases}

Acute myocardial infarction caused by thrombosis of left main coronary artery generate acute cardiac failure, cardiogenic shock and death. Along with the clinical history, the electrocardiogram (EKG) is the most useful tool for its recognition and timely management. Classically the EKG shows ST elevation $>1 \mathrm{~mm}$ in aVR or V1 with ST depression in the other leads. Urgent coronary angiography with percutaneous coronary angioplasty using drug eluting stents is recommended when the diagnosis is made. We report two cases to exemplify the clinical presentation, EKG and angiographic findings and therapeutic approach.

(Rev Med Chile 2020; 148: 1508-1512)

Key words: Coronary Artery Disease; Diagnosis; Electrocardiogram; Myocardial Infarction.
$\mathrm{E}$ 1 término infarto agudo al miocardio (IAM), se emplea cuando hay evidencia de daño miocárdico, definido como la elevación de Troponinas cardiacas $(\mathrm{Tnc})$ a valores superiores al percentil 99 del límite superior de referencia, con presencia de necrosis en contexto clínico de isquemia miocárdica, reflejada a lo menos por uno de los siguientes elementos: dolor, cambios del electrocardiograma (ECG), perdida de viabilidad miocárdica por imágenes y presencia de trombo intracoronario por angiografía ${ }^{1,2}$.

En el ECG, el IAM tipo I caracterizado principalmente por oclusión coronaria aterotrombótica, puede presentarse con elevación persistente del ST en dos derivaciones contiguas o con nuevo bloqueo de rama (IAMCEST); o también sin elevación del ST, con depresión aislada o difusa, transitoria o persistente, en múltiples derivadas $(\text { IAMSEST) })^{1,2}$.
En Guías Europeas del 2017 la mortalidad para el IAMCEST se estima en $4-12 \%$ y para el IAMSEST en $10 \%{ }^{2}$, constituyendo la ateroesclerosis (ATE) coronaria la causa más común.

En el IAM asociado a compromiso hemodinámico, la presencia de supradesnivel del ST $(\mathrm{SDST}) \geq$ de $1 \mathrm{~mm}$ en la derivación aVR y/o en $\mathrm{V} 1$ junto a un infradesnivel del ST (IDST) $\geq 1 \mathrm{~mm}$ en seis o más derivaciones, se debe plantear una obstrucción trombótica aguda del tronco común de coronaria izquierda (TCI) o enfermedad de múltiples vasos ${ }^{1,2}$.

El precoz reconocimiento clínico y por ECG del IAM por obstrucción del TCI es fundamental para un manejo adecuado y es en la actualidad una indicación urgente de coronariografía y revascularización miocárdica al igual que el IAMCEST ${ }^{1,2}$.

Comunicamos dos casos con IAM tipo I cuyo ECG es compatible con obstrucción del TCI con- 
firmada por coronariografía, tratados oportunamente y eficazmente. Los pacientes concedieron que sus casos fueran publicados.

\section{Caso 1}

Mujer de 74 años, diabética (DM) II, fumadora y dislipidémica, consultó a Urgencia por dolor opresivo retroesternal de varias horas de evolución, asociado a disnea, sudoración profusa y agitación psicomotora. Examen físico: desorientada, taquicárdica, polipneica, con hipoperfusión periférica y presión arterial 80/60. Auscultación cardíaca: ritmo regular en 2 tiempos sin soplos. Pulmones: estertores bibasales. ECG: ritmo sinusal (RS) $146 \mathrm{lpm}$, hemibloqueo izquierdo anterior (HBIA) e imagen de bloqueo completo de rama derecha (BCRD) con marcado SDST en aVR, e IDST en DII, DIII, y de V3 a V6, con ondas T negativas en V1 y V2. Laboratorio: Tnc I 9,07 ng/ml, $\mathrm{CK} / / \mathrm{MB} 409 \mathrm{u} / \mathrm{l} / / 7,86 \mathrm{ng} / \mathrm{ml}$. Gasometría arterial con $\mathrm{O}_{2}$ ambiental: $\mathrm{pH} 7,51, \mathrm{pO} 245 \mathrm{mmHg}$, $\mathrm{pCO}_{2}$ 22 mmHg, HCO3 17,6 mmol/l, saturación 78,2\%. Nitrógeno ureico $53 \mathrm{mg} / \mathrm{dl}$, creatinina 3,22 mg/dl, ácido láctico $76 \mathrm{mg} / \mathrm{dl}$, protrombina 51\%, GOT $1.368 \mathrm{u} / \mathrm{l}$ y GPT $857 \mathrm{u} / \mathrm{l}$. Rx tórax: congestión pulmonar bilateral. Ecocardiografía bidimensional (Eco2D): hipokinesia antero apical de ventrículo izquierdo (VI) con fracción de eyección (FEVI) severamente disminuida: 0,25. En shock cardiogénico complicado de insuficiencia respiratoria,falla renal y hepática, bajo noradrenalina, adrenalina, milrinona y vasopresina, se conecto a ventilador mecánico (VM) efectuándose coronariografía de urgencia bajo balón de contrapulsación aortica (BCPA). Demostramos obstrucción proximal (95\%) del TCI y lesión crítica ostial en arteria coronaria derecha (ACD). Se efectuó angioplastia percutánea (APC) del TCI con implante de stent fármaco activo (FA) y en ostium de ACD con implante de doble stent FA y metálico, con buen resultado angiográfico. ECG a $36 \mathrm{~h}$ post APC mostró regresión SDST en aVR, del HBIA y BCRD, y del IDST difuso, quedando con QS de V1 a V3. Tnc I y CK/MB máximos: > $50 \mathrm{ng} / \mathrm{ml} \mathrm{y}$ $2.466 \mathrm{u} / \mathrm{l} / / 181 \mathrm{ng} / \mathrm{ml}$.

ECG y coronariografía pre y post APC en Figura 1.

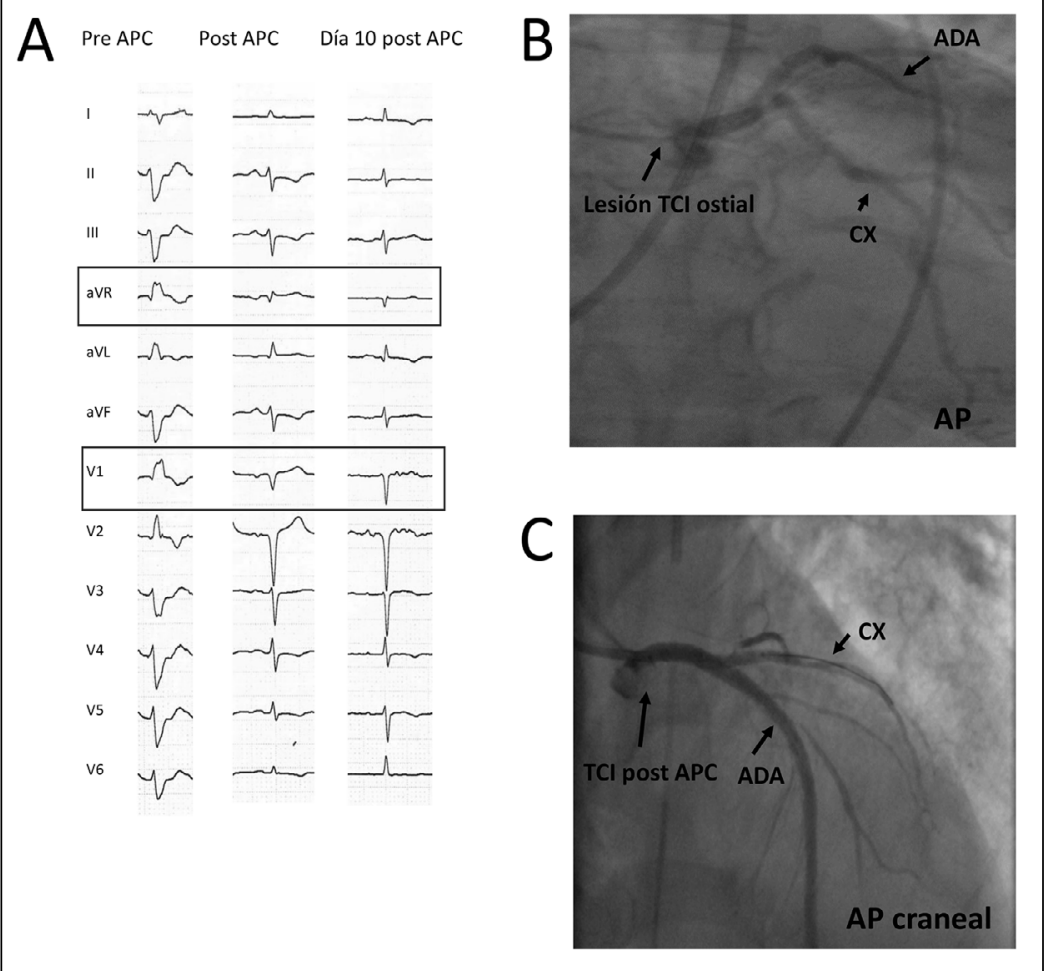

Figura 1. ECG y coronariografía pre y post angioplastia percutánea (APC). A: En relación con APC se observa evolución electrocardiográfica del supradesnivel ST en AVR y V1, con regresión del HBIA, BCRD, y del infradesnivel difuso del ST, con QS V1 a V3, a las $36 \mathrm{~h}$ y 10 días post $A P C$, respectivamente. $\mathbf{B}$ : Coronariografía pre APC. Estenosis de $95 \%$ de ostium proximal tronco coronario izquierdo (TCl). Arteria descendente anterior (ADA) y arteria circunfleja (ACX) sin lesiones. C: Coronariografía post APC. Resultado exitoso tras APC e implante de stent fármaco activo (FA) en $\mathrm{TCl}$. 
Permaneció en VM por 13 días con retiro progresivo de drogas vasoactivas, con doble antiagregación plaquetaria y estatinas. Alta día 25 con FEVI 0.35 por Eco 2D, en buenas condiciones, sin angina, arritmias, ni manifestaciones de insuficiencia cardíaca, con bisoprolol, losartan, furosemida y espironolactona.

\section{Caso 2}

Hombre de 55 años, consultó a Urgencia por dolor retroesternal opresivo, de pocas horas de evolución asociado a hipotensión 70/56, náuseas, con piel fría y sudorosa. ECG: RS $92 \mathrm{lpm}$, SDST en aVR, en V2 y V3, con onda T puntiaguda, e IDST en resto de derivaciones. Laboratorio: Tnc I 0,05 ng/ml, CK/MB 166 u/l//1,06 ng/ml. Gasometría arterial en $\mathrm{VM}$ con $\mathrm{O}_{2} 50 \%$ : $\mathrm{pH} 7,25, \mathrm{pO}_{2}$ 82,9, $\mathrm{pCO}_{2} 37,9, \mathrm{HCO} 316,2$, saturación 95,3\%. Nitrógeno ureico $23 \mathrm{mg} / \mathrm{dl}$, creatinina $1,25 \mathrm{mg} / \mathrm{dl}$, ácido láctico $77 \mathrm{mg} / \mathrm{dl}$, protrombina 41\%, GOT $845 \mathrm{u} / \mathrm{l}$ y GPT 210 u/l. Rx de tórax: congestión pulmonar bilateral. En shock cardiogénico bajo noradrena- lina y adrenalina, se efectuó coronariografía de urgencia en VM y BCPA. Demostramos lesión severa en porción distal de TCI, que comprometía origen de arteria descendente anterior (ADA) y de arteria circunfleja ( $\mathrm{ACx}$ ) efectuándose $\mathrm{APC}$ en TCI, implante de doble stent en ADA y otro en $\mathrm{ACx}$, todos $\mathrm{FA}$, con buen resultado angiográfico. ECG a una hora post APC mostró regresión SDST en aVR, de V1 a V3, y del IDST en resto de derivaciones, con $\mathrm{R}$ embrionaria en pared anterior. Tnc I y CK/MB máximos $>50 \mathrm{ng} / \mathrm{ml}$ y $10.099 \mathrm{u} / \mathrm{l} / />300$ $\mathrm{ng} / \mathrm{ml}$. A las $48 \mathrm{~h}$, Eco 2D mostró akinesia antero apical y lateral con FEVI de 0,5, evolucionando con insuficiencia cardiorrespiratoria, con apoyo de milrinona, dobutamina, nitroglicerina y furosemida, con doble antiagregación plaquetaria y estatinas, complicándose de neumonía y colecisto-pancreatitis aguda, manejada medicamente, en VM durante 10 días. Alta día 38, con FEVI 0,45 por Eco 2D, en buena condición cardiovascular, con bisoprolol, furosemida, atorvastatina, espironolactona e isosorbide.

ECG y coronariografía pre y post APC en Figura 2.

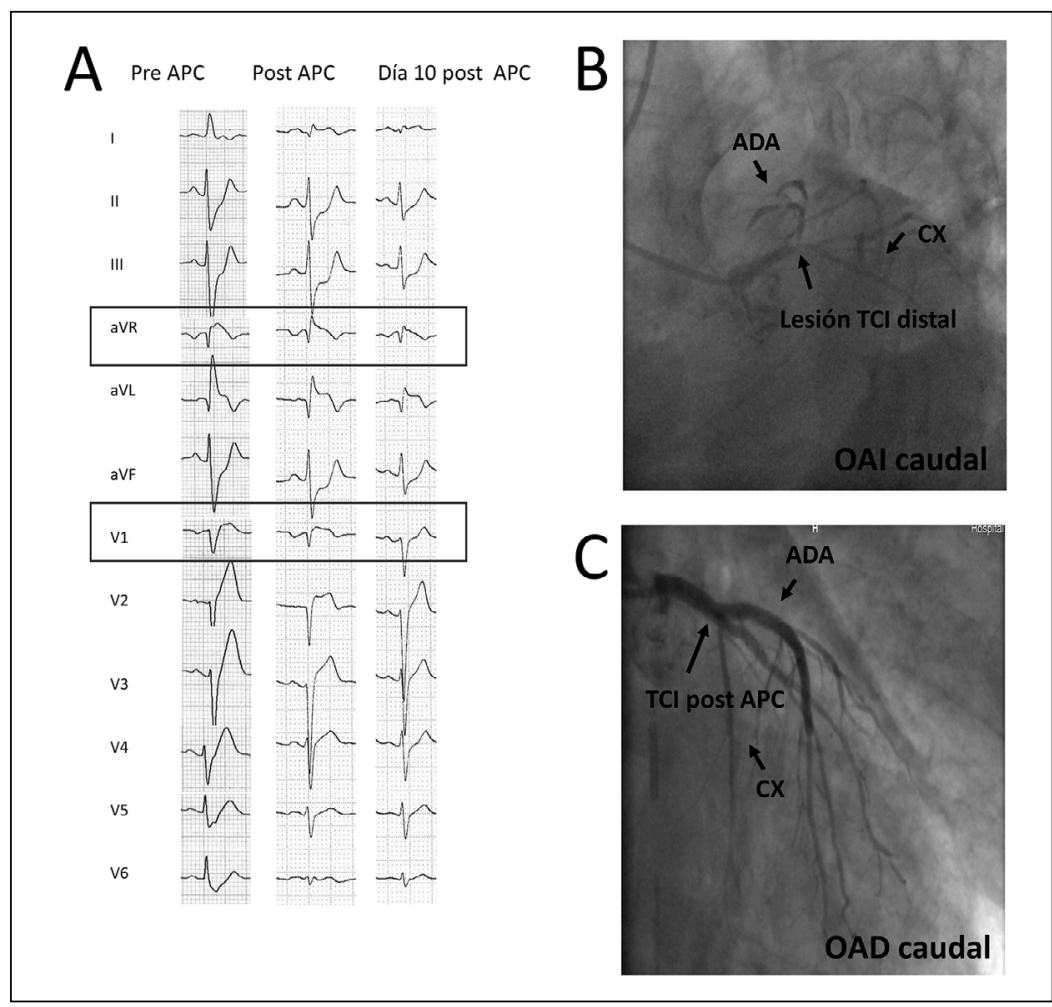

Figura 2. ECG y coronariografía pre y post angioplastia percutánea (APC). A: En relación con APC se observa evolución electrocardiográfica con regresión del supradesnivel del ST en aVR, de V1 a V3, de onda $T$ puntiaguda y del infradesnivel del ST en resto de derivaciones, quedando con R embrionaria en pared anterior, a la hora y décimo día post APC, respectivamente. B: Coronariografía pre APC. Estenosis severa en porción distal tronco coronario izquierdo (TCl) que compromete origen arteria descendente anterior (ADA) y arteria circunfleja (ACX). C: Coronariografía post APC. Resultado exitoso tras APC de $\mathrm{TCl}$ e implante de doble stent en ADA y uno en $A C x$, los tres FA. 


\section{Discusión}

Inventada por Goldberger en 1942, la derivación unipolar aVR del ECG con electrodo positivo en el brazo derecho fue ignorada por años al no visualizar una zona específica de VI. En la clínica, aVR adquiere gran importancia en el diagnóstico de la taquicardia ventricular, pericarditis aguda, síndrome de Brugada, bloqueo fascicular de rama derecha, taquicardia auricular izquierda y especialmente en la identificación de la arteria culposa del $\mathrm{IAM}^{3}$.

La derivación aVR explora el segmento basal del septum interventricular y tracto de salida de ventrículo derecho, por lo que frente a una lesión obstructiva hemodinámicamente significativa del TCI, el vector de lesión apunta hacia la derecha y arriba en el plano frontal, localizándose entre -90 y -180 grados, reflejandose al ECG en SDST en aVR y V1, con IDST en DII, DIII y de V4 a V6, complicándose frecuentemente con BCRD y/o bloqueos fasciculares de la rama izquierda ${ }^{4}$, ambos marcadores de severa isquemia miocárdica.

$\mathrm{La}$ incidencia de BCRD aislado en el IAM es de $6,3 \%$ y asociada a HBIA en $3,2 \%$, ambos determinados por obstrucción de ADA y ramas septales. Cuando el BCRD aparece de entrada en un IAM la incidencia de shock cardiogénico alcanza $15 \% .^{5}$

Casi 50\% de los IAM con BCRD no presentan SDST, estando presente una oclusión del TCI en $26-37 \%$ de los casos, situación clínica de alta mortalidad vinculada a extensa necrosis de pared anterior, insuficiencia cardíaca y shock cardiogénico ${ }^{6}$, entidad definida por hipotensión sistólica $<90 \mathrm{~mm} / \mathrm{Hg}$, con o sin necesidad del uso de catecolaminas, congestión pulmonar y signos de falla multiorgánica ${ }^{2,7}$, situación clínica presente en nuestros pacientes.

El mecanismo postulado para el SDST en AVR sería por isquemia subendocárdica difusa y global con IDST en derivaciones laterales con cambios recíprocos en aVR o por necrosis del septum basal. ${ }^{8}$

La sensibilidad del SDST en AVR para predecir una lesión obstructiva del TCI es de 54\%, con una especificidad de $90 \%$, con valor predictivo positivo de $71 \%$ y negativo de $80 \%$.

Cuando la relación entre el SDST en aVR y V1 es mayor a 1, permite diferenciar el compromiso de TCI frente ADA con sensibilidad y especificidad de $80 \%$.
En una serie de casos con IAMCEST con SDST en aVR se vio que $88 \%$ tenía lesión de TCI, $43 \%$ de ADA y $8 \%$ de ACD .

Con respecto al pronóstico, el SDST en aVR $\geq 1 \mathrm{~mm}$ se asocia a mayor mortalidad a 30 días en IAM, tanto de cara anterior $(23,5 \%$ con ST $\geq 1,5 \mathrm{~mm}$ y $11,5 \%$ con ST igual a $1 \mathrm{~mm}$ ), como de cara inferior $(22,5 \%$ con ST $\geq 1,5$ y $13,2 \%$ con ST igual a $1 \mathrm{~mm})^{9}$.

La enfermedad del TCI corresponde al 4-9\% de los pacientes referidos para coronariografía y desde el punto de vista clínico se puede presentar con angina estable (47-53\%), angina inestable (24-39\%), IAM (15\%) e isquemia silente $(7 \%){ }^{10}$

El TCI aporta el 84\% del flujo sanguíneo del VI en pacientes con circulación coronaria derecha dominante, de ahí que, si la obstrucción es crítica y se asocia a compromiso hemodinámico, la mortalidad alcanza el $70 \%{ }^{10}$.

Dentro de los predictores clínicos más importantes de una obstrucción angiográficamente significativa ( $>50 \%$ ) del TCI y que debuta con síndrome coronario agudo sin SDST, se señalan la edad $>65$ años, DM, insuficiencia renal, cardíaca y shock cardiogénico con una incidencia de estos últimos dos, de $71 \%$ y $21 \%$, respectivamente $^{11}$.

En el contexto del IAM, la isquemia miocárdica aguda es el evento que precede al shock cardiogénico en la mayoría de los pacientes sometidos a revascularización miocárdica ${ }^{12}$.

$\mathrm{Al}$ igual que en el IAMCEST, en el IAMSEST por obstrucción del TCI, la estrategia invasiva con coronariografía de urgencia $(<2 \mathrm{~h})$ vía arteria radial, seguida de APC e implante de stent FA, se ha convertido en el tratamiento estándar para pacientes con alto riesgo, que se presentan con dolor torácico persistente, insuficiencia cardíaca aguda y shock cardiogénico como fue el caso de nuestros pacientes, todo bajo soporte hemodinámico con drogas vasoactivas, y apoyo con $\mathrm{VM}^{2,12}$.

El BCPA no modifica el pronóstico y sobrevida del IAM complicado de shock cardiogénico en ausencia de complicaciones mecánicas ${ }^{2,7}$, pero en nuestra experiencia fue muy importante su utilización, considerando la extrema gravedad de ambos pacientes. La correcta interpretación del ECG más un buen diagnóstico clínico fueron fundamentales para el reconocimiento oportuno y manejo agresivo del IAM. 


\section{Referencias}

1. Thygesen K, Alpert J, Jaffe A, Chaitman B, Bax J, Morrow D, et al. Fourth Universal Definition of Myocardial Infarction. Eur Heart J 2019; 40: 237-69.

2. Ibañez B, James S, Agewall S, Antunes M, Bucciarelli-Ducci C, Bueno H, et al. 2017 ESC Guidelines for the Management of acute myocardial infarction in patients presenting with ST elevation. Eur Heart J 2018; 39: 11977.

3. Pérez A, Ferreira C, Ferreira FC, Dubner S, Barbosa R, Barbosa R, et al. Clinical Value of Lead aVR. Ann Noninvasive Electrocardiol 2011; 16 (3): 295-302.

4. Pérez A, Barbosa R. Clave diagnóstica de la obstrucción del tronco de la coronaria izquierda utilizando la dirección del vector de lesión. Rev Fed Arg Cardiol 2012; 41 (1).

5. Widimsky P, Rohac F, Stasek J, Kala P, Rokyta R, Kuzmanov B, et al. Primary angioplasty in acute myocardial infarction with right bundle branch block: should new onset of right bundle branch block be added to future guidelines as an indication for reperfusion therapy?. Eur Heart J 2012; 33: 86-95.

6. Gilliot G, Monney P Muller O, Muller O, Hugli O. Significance of an isolated bundle branch block in a patient with chest pain. BMJ Case Rep 2015. doi: 10.1136/bcr2015-209435.

7. Zeymer U, Bueno H, Granger CB, et al. Acute Cardiovascular Care Association position statement for the diagnosis and treatment of patients with acute myocardial infarction complicated by cardiogenic shock: A document of the Acute Cardiovascular Care Association of the European Society of Cardiology. Eur Heart J Acute Cardiovasc Care 2020; 9 (2): 183-97. doi:10.1177/2048872619894254.

8. Sen F, Ozeke O, Kirbas O, Burak C, Kafes H, Tekin Tak $\mathrm{B}$, et al. Classical electrocardiographic clues for left main coronary artery disease. Indian Heart J 2016; 68: 5226-7.

9. Medina F, Barreto A, Rosales A, Rosales A, Rojas Gino Román R, et al. Supradesnivel del segmento ST en derivación aVR en el síndrome coronario agudo con elevación persistente del segmento ST. Revisión de la literatura a propósito de un caso. Rev Med Hered 2016; 27: 106-10.

10. Collet C, Capodanno D, Onuma Y, Banning A, Stone G, Taggart D, et al. Left main artery disease: pathophysiology, diagnosis, and treatment. Nature Reviews Cardiology 2018; 15 (6): 321-33.

11. Claver E, Curós A, López-Ayerbe J, Serra J, Mauri J, Fernández-Nofrerías E, et al. et al. Clinical Predictors of Left Main Coronary Artery Disease in High-Risk Patients With a First Episode of Non-St-Segment Elevation Acute Coronary Syndrome. Rev Esp Cardiol 2006; 59 (8): 794-800.

12. Neuman FJ, Sousa-Uva M, Ahlson A, Alfonso F, Banning A, Benedetto H. et al. Guía ESC/EACTS 2018 sobre revascularización miocárdica. Rev Esp Cardiol 2019; 72 (1): 73. e1-76. 